Service contribution and cost-effectiveness of specialist registrars in NHS trusts: a survey and costing analysis

Editor - The recent paper by Dafydd et al ${ }^{1}$ is a welcome attempt to quantify the service contribution of specialist registrars. However, the cost calculation is more complex. The paper focuses more on financial modelling for surgical registrars; independent operations and high volume outpatient clinic consultations lend themselves more easily to cost analysis compared with less interventional medical specialties, eg endocrinology or elderly care medicine.

In contrast to high volume surgical/orthopaedic clinics, outpatient clinics in many of the medical specialties may require at least 30 minutes for new patients and 15 minutes for follow-up patients (significantly longer in some specialist clinics), allowing registrars to see a maximum of about 9 patients per clinic, without necessarily attracting a higher tariff. Other additional factors to medical registrars' time in clinic include on call duties, annual/study leave, regional training days and inpatient specialty consultations. A recent audit of ten respiratory specialist registrars at a major teaching hospital revealed a mean 38.4 respiratory clinics attended per year (or just below 0.75 clinics per week) despite them being rostered consistently for two clinics per week when available to be listed (unpublished observations), equating to less than seven outpatients per week or approximately $£ 55,000$ per year (assuming a 50:50 mix of new patients and follow-ups).

Procedure lists for respiratory trainees are affected by changes in training, eg $31.4 \%$ attrition rate in bronchoscopy procedures following the European Working Time Directive or 65 per year equating to $£ 11,375$ per year (assuming $50 \%$ of procedures are independent and $£ 350$ per bronchoscopy). ${ }^{2}$ In total, this example estimates $\mathfrak{E} 66,375$ per year for a respiratory registrar compared with $\mathfrak{£} 700,000$ for a surgical registrar. How to compare value though? Moreover, this does not include inpatient specialty referrals, ambulatory care consults or other procedures (eg chest drains, indwelling pleural catheters, thoracoscopy or EBUS-TBNA) that might attract higher tariffs, ${ }^{3}$ or contribution to the acute medical take,so the calculation becomes ever more complex. What about the quality of service contribution not just the quantity?

In summary, attempting to quantify service contribution by specialist registrars in the NHS is laudable but the calculations and quality metrics are complex, especially for specialist physicians in training in less interventional specialties. What are the best markers: patient quality of life measures, patient/relatives complaints/compliments, time taken/number of tests to get the correct diagnosis? Until such time as these markers are identified, costs will tend to favour surgical/interventional specialty registrars but further work is needed in this area.

\section{Conflicts of interest}

The author has no conflicts of interest to declare.

ANDREW RL MEDFORD

consultant and honorary senior lecturer in respiratory medicine, north Bristol Lung Centre, Southmead Hospital, Bristol, UK

\section{References}

1 Dafydd DA, Baskaradas A, Bobdiwala S et al. Service contribution and cost-effectiveness of specialist registrars in NHS trusts: a survey and costing analysis. Clin Med 2016;16:235-9.

2 Medford AR. Impact of the European Working Time Directive on specialty training. Qual Saf Health Care 2008;17:79-80.

3 Medford AR. Aberrant clinical coding: an underappreciated entity for NHS Trusts, clinicians and coders. J R Coll Physicians Edinb 2013;43:101-2.

\section{Assessment for benign paroxysmal positioning vertigo in medical patients admitted with falls in a district general hospital}

Editor - Abbott et al imply that benign paroxysmal positioning vertigo (BPPV) may be overlooked in patients admitted to a district general hospital with falls and, ipsi facto, that this condition might be the cause of such falls. This cannot be justified on the basis of the data presented in this paper.

Although the authors describe in their introduction the diagnostic criteria for BPPV, to my surprise, in their short results section they make no mention of whether (a) the patient actually had the characteristic symptoms of BPPV, as opposed to falls alone; or (b) the characteristic pattern of nystagmus was elicited during the provocative Dix-Hallpike manoeuvre (that is, a torsional and horizontal nystagmus beating towards the lower ear, which develops after a latency of a few seconds, persists for $10-15$ seconds and is accompanied by vertigo ${ }^{2}$ ); nor did they include laterality or which semi-circular canals were implicated in the falls. They simply state that ' 20 were positive for BPPV (54\%) and 17 patients were negative'.

Abbott et al did not consider that the falls were the cause of any BPPV. By way of example, a few days ago I examined an older patient with a minor head injury after a fall in his bathroom who began the history of their dizziness, which developed soon thereafter, with: 'doctor, when I roll over in bed...'- they hardly need go on and sure enough have typical neuro-opthalmic findings of BPPV referable to the left side. This relationship to injury is a common observation in officebased practice.

Over recent years, BPPV has become a well-recognised entity but practitioners tend to apply this manoeuvre in situations where this is inappropriate, such as an acute vestibulopathy (eg vestibular neuronitis), and where the patient is acutely dizzy and provocative head manoeuvres serve only to make the patient feel worse. This does not equate to BPPV caused by cupulolithiasis.

Although they describe the Epley manoeuvre particle repositioning manoeuvre, the authors did not provide any information as to whether their patients with falls were assisted by this.

More information is essential for readers of your journal to accept these authors' recommendation that 'all patients admitted with falls are assessed for BPPV to minimise their risk of further falls'.

\section{Conflicts of interest}

The author has no conflicts of interest to declare.

ANDREW M CHANCELLOR neurologist, Tauranga Hospital, Tauranga, New Zealand 


\section{References}

1 Abbott J, Tomassen S, Lane L, Bishop K, Thomas N. Assessment for benign paroxysmal positional vertigo in medical patients admitted with falls in a district general hospital. Clin Med 2016;16:335-8.

2 Halmagyi GM and Cremer PD. Assessment and treatment of dizziness. J Neurol Neurosurg Psychiatry 2000;68:129-34.

\section{Response}

Editor - We thank the correspondent for their letter regarding our recent article. ${ }^{1}$ We acknowledge the limitations to our study and its position as a pilot study, with wider plans for larger scale research in the area. In the first instance, the paper was submitted for publication in Clinical Medicine to highlight a possible gap and subsequently improve clinical practice.

It is well known that most falls are multifactorial and, furthermore, that benign paroxysmal positional vertigo (BPPV) significantly increases a patient's risk of falling. ${ }^{2}$ As the correspondent's letter stated, we cannot assume causality simply by the presence of BPPV, nor can we discern whether the BPPV had been caused by a head injury as a result of the fall.

In our article, we drew no conclusions regarding the specific causality because we did not have data regarding preceding symptomology and, furthermore, are aware of the often complex presentation in older adults with absence of classical symptoms. ${ }^{3}$

Similarly, we did not have the resources to do post-treatment surveillance. The aim of this study was not to determine whether BPPV was the cause of falls in this group but simply to establish the prevalence of BPPV in older adults admitted to hospital with falls.
Regardless of whether the diagnosis of BPPV was simply an incidental finding irrelevant to the cause of the fall, or indeed caused by the trauma of the admitting fall, it has been well established that treating BPPV reduces a person's risk of falls. ${ }^{2}$ As we have stated in our 'key points', a diagnosis of BPPV in a patient with known falls will hopefully reduce the risk of future falls following successful treatment.

We welcome the correspondent's opinion that more information is required within this field and we are currently drawing up plans to expand the scope of our team's research into BPPV.

\section{Conflicts of interest}

The author has no conflicts of interest to declare.

JOEL ABBOTT

geriatric medicine specialty registrar, Wrexham Maelor Hospital, Wrexham, UK

\section{References}

1 Abbott J, Tomassen S, Lane L, Bishop K, Thomas N. Assessment for benign paroxysmal positional vertigo in medical patients admitted with falls in a district general hospital. Clin Med 2016;16:335-8.

2 Gananca FF, Gazzola JM, Gananca CF. Elderly falls associated with benign paroxysmal positional vertigo. Braz J Otorhinolaryngol 2010;76:113-20.

3 Pollak L. Awareness of benign paroxysmal positional vertigo in central Isreal. BMC Neurol 2009;9:17.

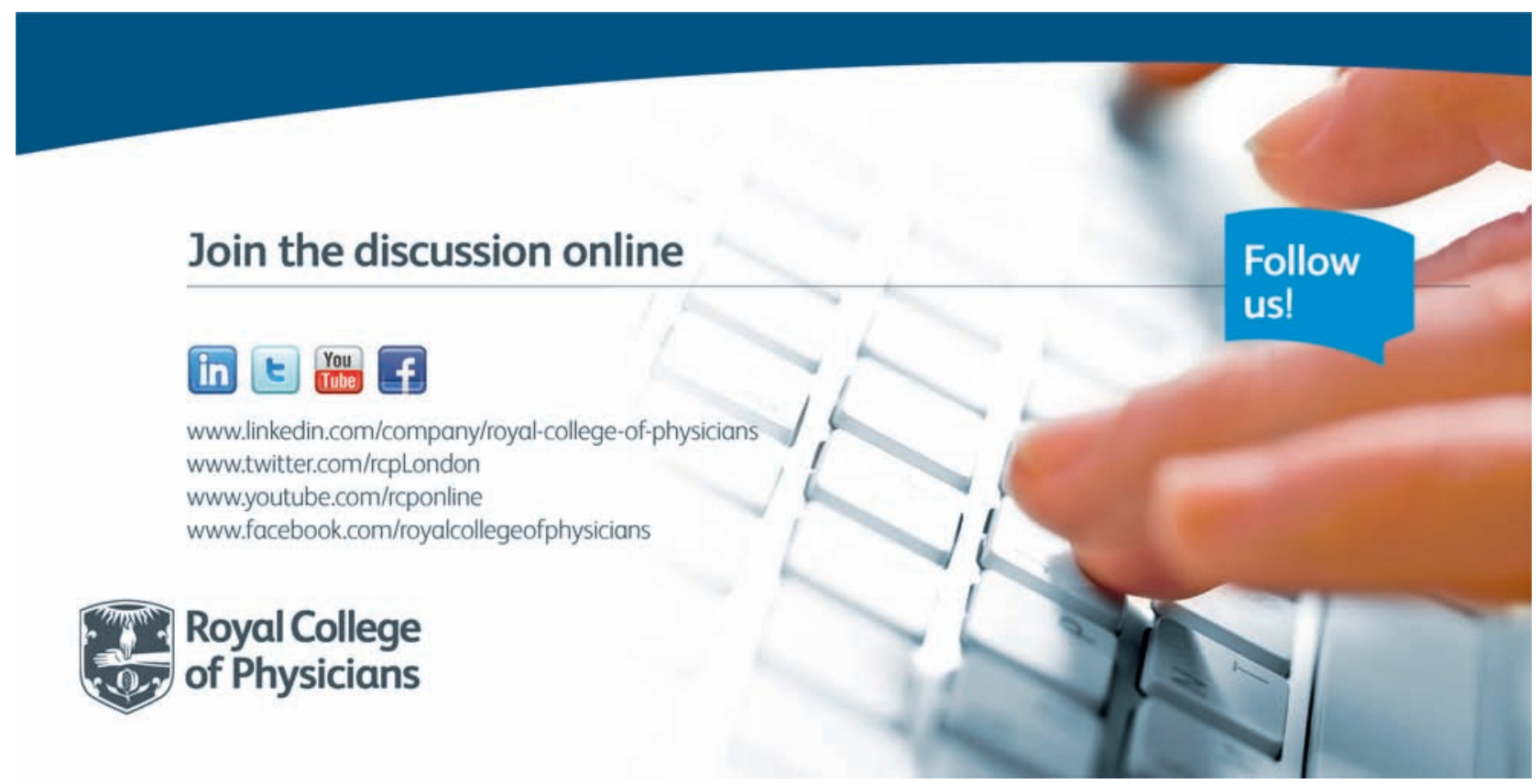

